\title{
Endocytosis of adiponectin receptor 1 through a clathrin- and Rab5-dependent pathway
}

\author{
Qiurong Ding ${ }^{1}$, Zhenzhen Wang ${ }^{1}$, Yan Chen ${ }^{1}$ \\ ${ }^{1}$ Key Laboratory of Nutrition and Metabolism, Institute for Nutritional Sciences, Shanghai Institutes for Biological Sciences, Chinese \\ Academy of Sciences, Graduate School of the Chinese Academy of Sciences, 294 Taiyuan Road, Shanghai 200031, China
}

\begin{abstract}
In eukaryotic cells, receptor endocytosis is a key event regulating signaling transduction. Adiponectin receptors belong to a new receptor family that is distinct from G-protein-coupled receptors and has critical roles in the pathogenesis of diabetes and metabolic syndrome. Here, we analyzed the endocytosis of adiponectin and adiponectin receptor 1 (AdipoR1) and found that they are both internalized into transferrin-positive compartments that follow similar traffic routes. Blocking clathrin-mediated endocytosis by expressing Eps15 mutants or depleting $\mathrm{K}^{+}$trapped AdipoR1 at the plasma membrane, and $\mathrm{K}^{+}$depletion abolished adiponectin internalization, indicating that the endocytosis of AdipoR1 and adiponectin is clathrin-dependent. Depletion of $\mathrm{K}^{+}$and overexpression of Eps15 mutants enhance adiponectinstimulated AMP-activated protein kinase phosphorylation, suggesting that the endocytosis of AdipoR1 might downregulate adiponectin signaling. In addition, AdipoR1 colocalizes with the small GTPase Rab5, and a dominant negative Rab5 abrogates AdipoR1 endocytosis. These data indicate that AdipoR1 is internalized through a clathrin- and Rab5dependent pathway and that endocytosis may play a role in the regulation of adiponectin signaling.
\end{abstract}

Keywords: adiponectin, adiponectin receptors, clathrin, endocytosis, Rab5

Cell Research (2009) 19:317-327. doi: 10.1038/cr.2008.299; published online 4 November 2008

\section{Introduction}

The prevalence of type 2 diabetes has increased dramatically in recent years. White adipose tissue (WAT) is a major site of energy storage that is important for energy homeostasis [1]. Recently, WAT has been increasingly recognized as an important endocrine organ that secretes many biologically functional adipokines. Of these adipokines, adiponectin has attracted the majority of attention because it displays positive antidiabetic and antiatherogenic effects and is expected to be a novel therapeutic tool for diabetes and metabolic syndrome. Adiponectin stimulates glucose utilization and fatty-acid combustion. Its antidiabetic function acts mainly through the activation of AMP-activated protein kinase (AMPK), which is a central regulator of intracellular energy metabolism [2].

Adiponectin exerts its protective roles by interacting with two membrane adiponectin receptors, AdipoR1

Correspondence: Yan Chen

Tel: +86-21-54920916; Fax: +86-21-54920291

E-mail: ychen3@sibs.ac.cn

Received 16 January 2008; revised 13 May 2008; accepted 14 July 2008; published online 4 November 2008 (adiponectin receptor 1) and AdipoR2 [3]. The functional significance of AdipoR1 and AdipoR2 in glucose metabolism has been demonstrated by gene deletion experiments, in which the knockout of both receptors was shown to be associated with insulin resistance and marked glucose intolerance [4]. AdipoR1 and AdipoR2 share 66.7\% identity in amino acid sequence; they are predicted to contain seven transmembrane domains that are topologically distinct from classical G-protein-coupled receptors (GPCRs). AdipoR1 is ubiquitously expressed, with abundant expression in the skeletal muscles, whereas AdipoR2 is most abundantly expressed in the liver. The activation of both AdipoR1 and AdipoR 2 by adiponectin binding stimulates AMPK activity; this regulation is associated with an increase in fattyacid oxidation and glucose uptake [3]. In addition, AdipoR1 and AdipoR2 have distinct functional signaling preferences. AdipoR1 is more prominent in AMPK phosphorylation, while AdipoR2 is more involved in PPAR $\alpha$ regulation [5]. How the signals are transduced from the adiponectin receptors to AMPK and how this process is regulated remain largely unknown. Recently, APPL1 was identified as an adaptor that interacts with the N-terminus of AdipoR1 [6]. The interaction of APPL1 with AdipoR1 is stimulated by adiponectin; this interaction plays an important role in 
downstream signaling, including AMPK phosphorylation, glucose uptake and lipid oxidation [6].

In eukaryotic cells, the internalization of membrane receptors, with or without ligand stimulation, has been proven to be an effective way to regulate downstream signaling. Most GPCRs are regulated by endocytosis [79]. The $\beta 2$-adrenergic receptor $\left(\beta_{2} \mathrm{AR}\right)$ was the first GPCR found to be regulated by internalization [7]. In response to agonist stimulation, $\beta_{2} \mathrm{AR}$ is internalized through a clathrin-mediated pathway and then downregulated via degradation in the lysosome. $\beta$-Arrestin acts as a clathrin adaptor that regulates the endocytosis of $\beta_{2} \mathrm{AR}[10,11]$. Many other types of membrane receptors are regulated by different endocytosis pathways. For example, activated EGF receptor (EGFR) recruits the $\mathrm{SH} 2$ domain-containing signaling molecule Grb2 (growth factor receptor-binding protein2), which mediates the binding of EGFR to the E3 ubiquitin ligase $\mathrm{c}-\mathrm{Cbl}$ and initiates a cascade of events leading to internalization and degradation of EGFR [12]. In contrast to $\beta_{2} \mathrm{AR}$ and EGFR, however, TGF- $\beta$ type II receptor internalization is constitutive and independent of ligand binding [13]. The clathrin-dependent endocytosis of the TGF- $\beta$ type II receptor is important for TGF- $\beta$-induced Smad activation and signaling transduction $[14,15]$.

The endocytosis of membrane receptors is mediated mainly by two pathways: a clathrin-dependent pathway and a clathrin-independent pathway; the latter is mediated mainly by caveolae and lipid rafts. In clathrin-mediated internalization, receptors are targeted to the early endosomes and are usually downregulated through an ubiquitindependent sorting process [16-18]. Moreover, the same receptor can be endocytosed through different pathways that lead to divergent signaling events. For example, TGF- $\beta$ receptors can undergo clathrin-mediated and caveolinmediated endocytosis. TGF- $\beta$ receptors that are internalized through clathrin are routed to EEA1-postive endosomes, thus promoting TGF- $\beta$ signaling. In contrast, the caveolindependent endocytosis of TGF- $\beta$ receptors leads mainly to rapid receptor turnover [14]. Therefore, the regulation of receptors through different internalization pathways is a critical way to modulate downstream signaling.

Due to the key role of adiponectin in the regulation of glucose and lipid metabolism, it is of great importance to understand the regulation of adiponectin signaling at various levels. It is not yet known how adiponectin and adiponectin receptors are regulated by endocytosis. Here, we provide an initial characterization of the endocytosis of adiponectin and AdipoR1. Our study suggests that adiponectin and AdipoR1 are internalized through a clathrin-mediated pathway involving Rab5. In addition, we found that inhibition of AdipoR1 endocytosis enhances adiponectin-induced AMPK phosphorylation, suggesting that endocytosis may play a role in the regulation of adiponectin signaling.

\section{Results}

\section{Endocytosis of adiponectin}

We first analyzed the endocytosis of full-length adiponectin in Hela cells. Recombinant human adiponectin was labeled with Alexa-555. The cells were serum-starved for $24 \mathrm{~h}$ and then incubated with $10 \mu \mathrm{g} / \mathrm{ml}$ of labeled full-length adiponectin for $2 \mathrm{~h}$. The amount of adiponectin used was consistent with its physiological concentration [19]. After incubation, the cells were washed to remove the surface-bound adiponectin. As shown in Figure 1A, we observed obvious internalization of the labeled adiponectin. To confirm the specificity of the adiponectin internalization, a 20 -fold excess of unlabeled adiponectin was added to the medium. We found that endocytosis of the labeled adiponectin was dramatically decreased (Figure 1A, right panel). These results provide the first piece of evidence that adiponectin could be specifically endocytosed into these cells.

\section{Endocytosis of AdipoR1}

Adiponectin receptors belong to a new seven-transmembrane receptor family termed PAQR; these receptors have the N-terminus facing the cytosol, unlike GPCRs [3, 20]. In previous experiments, we found that, when overexpressed in Hela cells, AdipoR1 is distributed in the cytoplasm with a punctate pattern and AdipoR2 is dispersed in the cytosol without obvious puncta (data not shown), indicating that AdipoR1 could be localized both in the plasma membrane and in the cytosol. To analyze only the plasma membranelocalized receptors, we generated C-terminally Myc-tagged AdipoR1 and AdipoR2 and used them to analyze endocytosis in HEK293T cells, a cell line commonly used to observe receptor endocytosis. The C-termini of adiponectin receptors are proposed to localize to the extracellular side of the plasma membrane $[3,20]$. The tagged receptors on the cell surface were labeled at $4{ }^{\circ} \mathrm{C}$ with an anti-Myc antibody and a fluorescently conjugated secondary antibody. Consistent with the topology of AdipoR1 that was shown by Yamauchi et al.[3], we found a clear plasma membrane localization of AdipoR1 (Figure 1B, first panel); however, we failed to detect membrane-bound AdipoR2 in HEK293T cells, possibly due to insignificant plasma membrane localization of AdipoR2. Therefore, we focused on AdipoR1 in subsequent experiments. After the cells were serum starved overnight, the endocytosis of AdipoR1 was analyzed by incubating at $37^{\circ} \mathrm{C}$ in complete medium for different periods of time. At 5 min after incubation, it appeared that some of the membrane receptors began to be internalized. At 30 and $60 \mathrm{~min}$, most of the membrane receptors were internalized (Figure 1B). 

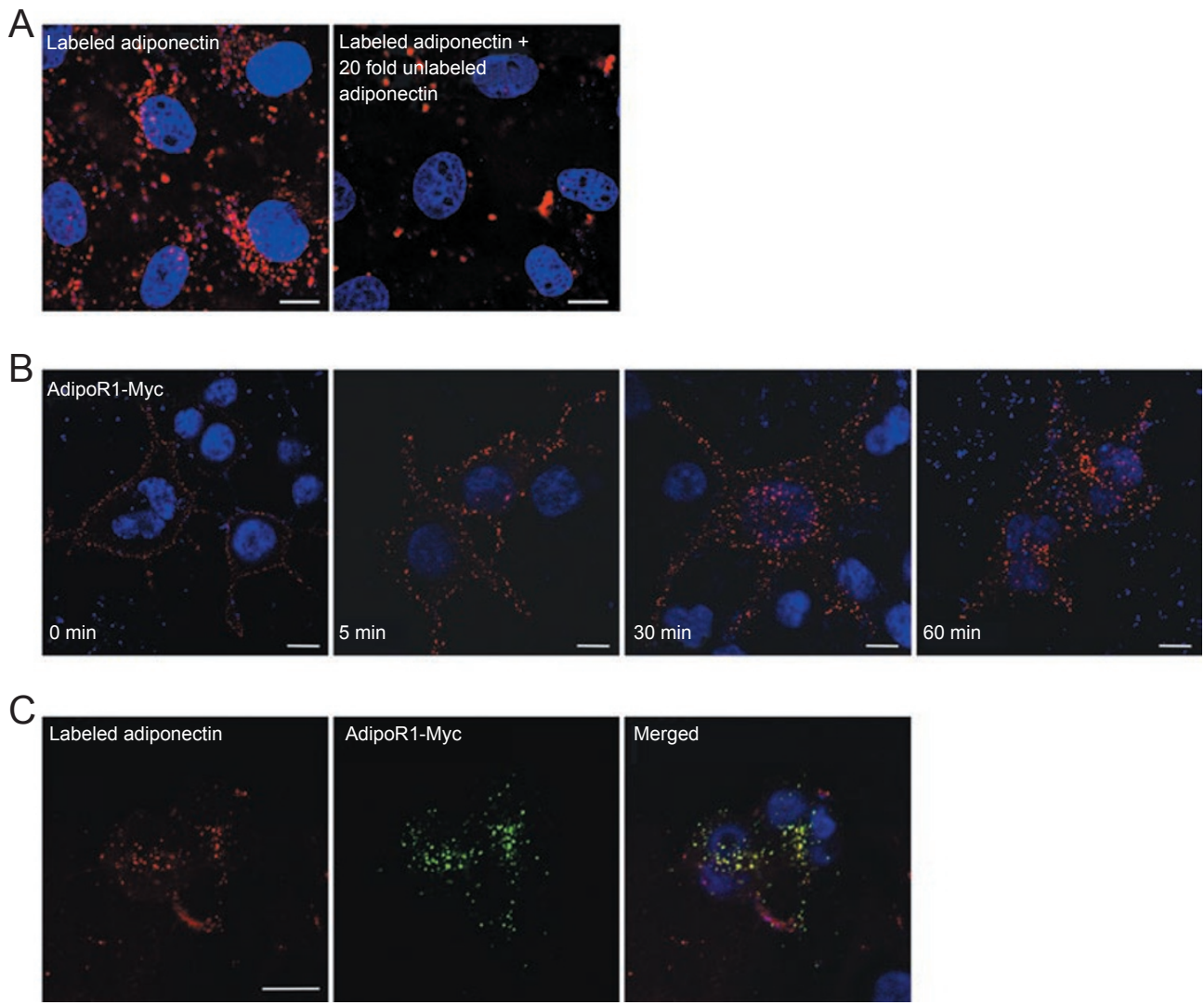

Figure 1 Endocytosis of adiponectin and AdipoR1. (A) Endocytosis of adiponectin. Recombinant human adiponectin was labeled with Alexa-555 (red). Hela cells were serum-starved for $24 \mathrm{~h}$ and then incubated with $10 \mu \mathrm{g} / \mathrm{ml}$ of labeled adiponectin for $2 \mathrm{~h}$ in the absence or presence of $200 \mu \mathrm{g} / \mathrm{ml}$ of unlabeled adiponectin. The cells were washed to remove the surface-bound adiponectin. The nuclei were stained with DAPI (blue). The cells were fixed and imaged by confocal microscopy. The scale bar indicates $10 \mu \mathrm{m}$. (B) Constitutive endocytosis of AdipoR1. HEK293T cells were transfected with Myc-tagged AdipoR1. At $48 \mathrm{~h}$ after transfection, the live cells were labeled at $4{ }^{\circ} \mathrm{C}$ with an anti-Myc antibody and a secondary antibody (red). After washing, the cells were then incubated at $37^{\circ} \mathrm{C}$ in complete medium for various times as indicated. The scale bar indicates $10 \mu \mathrm{m}$. (C) Adiponectin and AdipoR1 follow similar traffic routes during internalization. HEK293T cells were transfected with Myc-tagged AdipoR1 and serum-starved overnight. Membrane-bound AdipoR1 was labeled by immunostaining (green) at $4^{\circ} \mathrm{C}$. The cells were then incubated with labeled adiponectin (red) for $30 \mathrm{~min}$ at $37^{\circ} \mathrm{C}$. The scale bar indicates $10 \mu \mathrm{m}$.

To further confirm the endocytosis of AdipoR1, we used a different protocol to analyze only the membrane-localized but not internalized receptors. Consistent with our previous observations, the signal from the membrane-localized receptors before the initiation of endocytosis was much stronger than those at 5 or $30 \mathrm{~min}$ after endocytosis was started (Supplementary information, Figure S1).

We next examined whether or not the internalization of AdipoR1 was ligand-dependent. After serum starvation of the cells overnight, plasma membrane-bound AdipoR1 was labeled, and its internalization was initiated with or without the addition of $10 \mu \mathrm{g} / \mathrm{ml}$ adiponectin in the DMEM medium. We found little change in the endocytosis of AdipoR1 in the presence or absence of adiponectin (data not shown), indicating that AdipoR1 is constitutively endocytosed, independently of ligand stimulation, in HEK293T cells.
To further investigate whether adiponectin and AdipoR1 are internalized through similar traffic routes, we simultaneously examined the endocytosis of Alexa 555-labelled adiponectin and Myc-tagged AdipoR1 in HEK293T cells. The plasma membrane-bound AdipoR1 was labeled at $4{ }^{\circ} \mathrm{C}$; then the cells were incubated with $10 \mu \mathrm{g} / \mathrm{ml}$ of labeled adiponectin at $37^{\circ} \mathrm{C}$ for $30 \mathrm{~min}$ to allow for the internalization of adiponectin and AdipoR1. We found that the labeled adiponectin and AdipoR 1 were prominently colocalized in these cells (Figure 1C), indicating that adiponectin and AdipoR1 follow similar traffic routes during endocytosis.

Adiponectin and AdipoR1 are both internalized to transferrin-positive compartments

The endocytosis of membrane receptors occurs through two main pathways: clathrin-dependent and clathrin- 
independent. To find out which pathway is responsible for the internalization of adiponectin and AdipoR1, we used transferrin as a marker for the clathrin-mediated pathway and caveolin as a marker for the caveolin-mediated pathway, which constitutes the major clathrin-independent pathway $[21,22]$. We analyzed the endocytosis of full-length adiponectin in $\mathrm{C} 2 \mathrm{C} 12$ myotubes, as skeletal muscle is one of the major target organs of adiponectin and abundantly expresses AdipoR1 [2]. The differentiated $\mathrm{C} 2 \mathrm{C} 12$ cells were serum-starved for $2 \mathrm{~h}$ and then incubated with Alexa 488-labeled transferrin and Alexa 555-labeled adiponectin simultaneously. After $2 \mathrm{~h}$ of endocytosis, we found that transferrin and adiponectin were profoundly colocalized (Figure 2A). These data indicate that adiponectin is inter- nalized to a transferrin-positive compartment. To further investigate this phenomenon, we analyzed the movement of adiponectin at $10 \mathrm{~min}$ after the initiation of endocytosis. In addition, the transferrin receptor was also labeled as another marker for clathrin-mediated endocytosis. As shown in Figure 2B, we found that adiponectin colocalizes well with both transferrin and transferrin receptors. Meanwhile, we found that adiponectin has little colocalization with caveolin (Figure 2C). Taken together, our findings suggest that adiponectin is endocytosed in a clathrin-mediated pathway. Because both full-length and globular adiponectin can serve as ligands for adiponectin receptors [3], we also analyzed the internalization of globular adiponectin. We found that globular adiponectin could be internalized
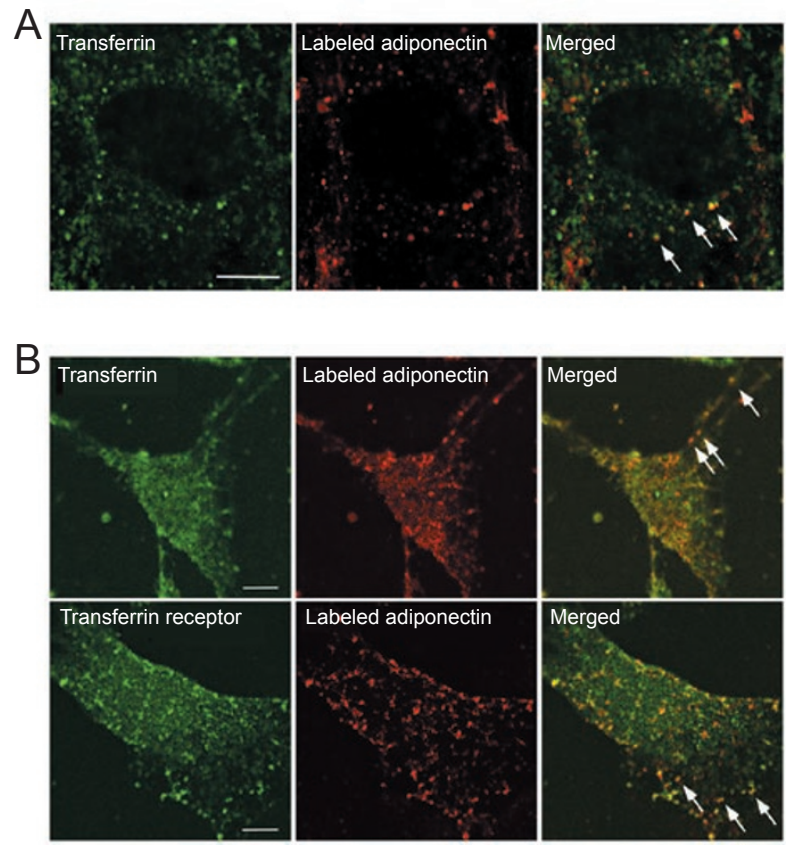
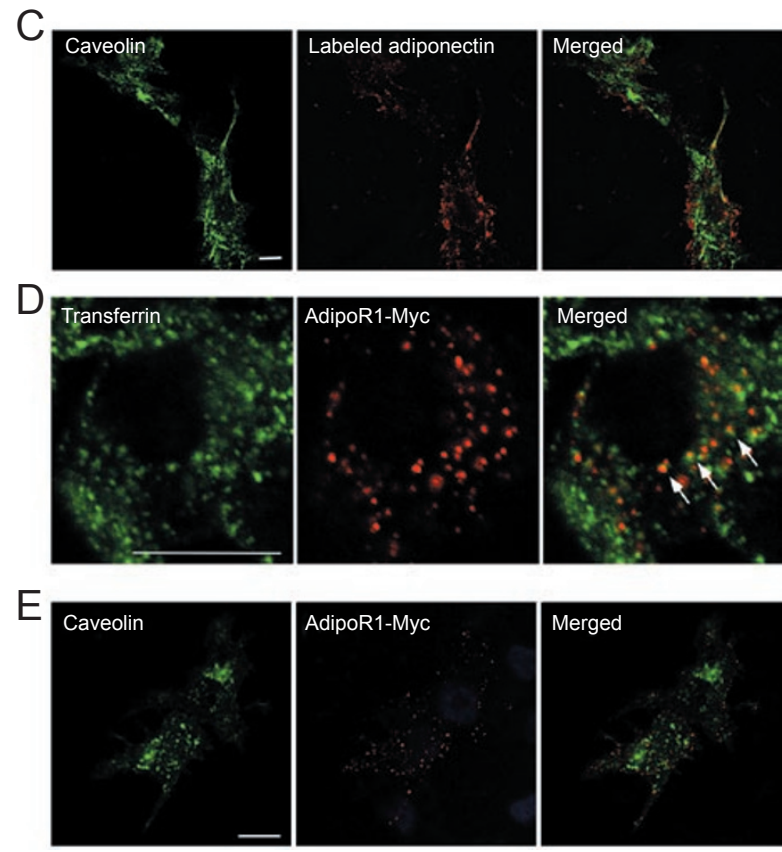

Figure 2 Adiponectin and AdipoR1 are both internalized into transferrin-positive compartments. (A) Endocytosis of adiponectin into transferrin-positive compartments. C2C12 cells were differentiated, serum-starved for $2 \mathrm{~h}$ and incubated with $50 \mu \mathrm{g} / \mathrm{ml}$ of labeled transferrin (green) and $10 \mu \mathrm{g} / \mathrm{ml}$ of labeled adiponectin (red) for $2 \mathrm{~h}$. The cells were washed to remove membranebound transferrin and adiponectin before analysis by confocal microscopy. The arrows indicate representative colocalization signals. The scale bar indicates $10 \mu \mathrm{m}$. (B) Colocalization studies of adiponectin with transferrin or transferrin receptors at 10 min after the initiation of endocytosis in differentiated $\mathrm{C} 2 \mathrm{C} 12$ cells. To study colocalization with transferrin receptors, cells were immunostained with an antibody specific for the transferrin receptor, followed by Alexa 488-conjugated secondary antibodies (green). The arrows indicate representative colocalization signals. The scale bar indicates $10 \mu \mathrm{m}$. (C) Colocalization studies of adiponectin with caveolin. Differentiated C2C12 cells were serum-starved for $2 \mathrm{~h}$ and then incubated with $10 \mu \mathrm{g} / \mathrm{ml}$ of labeled adiponectin (red) for $2 \mathrm{~h}$. The cells were immunostained with an anti-caveolin-1 antibody followed by an Alexa 488-conjugated secondary antibody (green). The scale bar indicates $10 \mu \mathrm{m}$. (D) AdipoR1 is internalized into transferrin-positive compartments. HEK293T cells were transfected with Myc-tagged AdipoR1. Membrane-bound AdipoR1 was labeled by immunostaining (red) at $4{ }^{\circ} \mathrm{C}$ before incubating with transferrin. The labeled AdipoR1 and transferrin (green) were subjected to internalization for 10 min at $37^{\circ} \mathrm{C}$ before fixation and confocal imaging. The arrows indicate representative colocalization of transferrin with AdipoR1. The scale bar indicates $10 \mu \mathrm{m}$. (E) AdipoR1 is not internalized into caveolin-positive compartments. HEK293T cells were transfected with Myc-tagged AdipoR1. Membrane-bound AdipoR1 was labeled by immunostaining (red) at $4{ }^{\circ} \mathrm{C}$ before internalization for $10 \mathrm{~min}$ at $37^{\circ} \mathrm{C}$. The cells were immunostained with an anti-caveolin-1 antibody followed by Alexa 488-conjugated secondary antibodies (green) after fixation. The scale bar indicates $10 \mu \mathrm{m}$. 

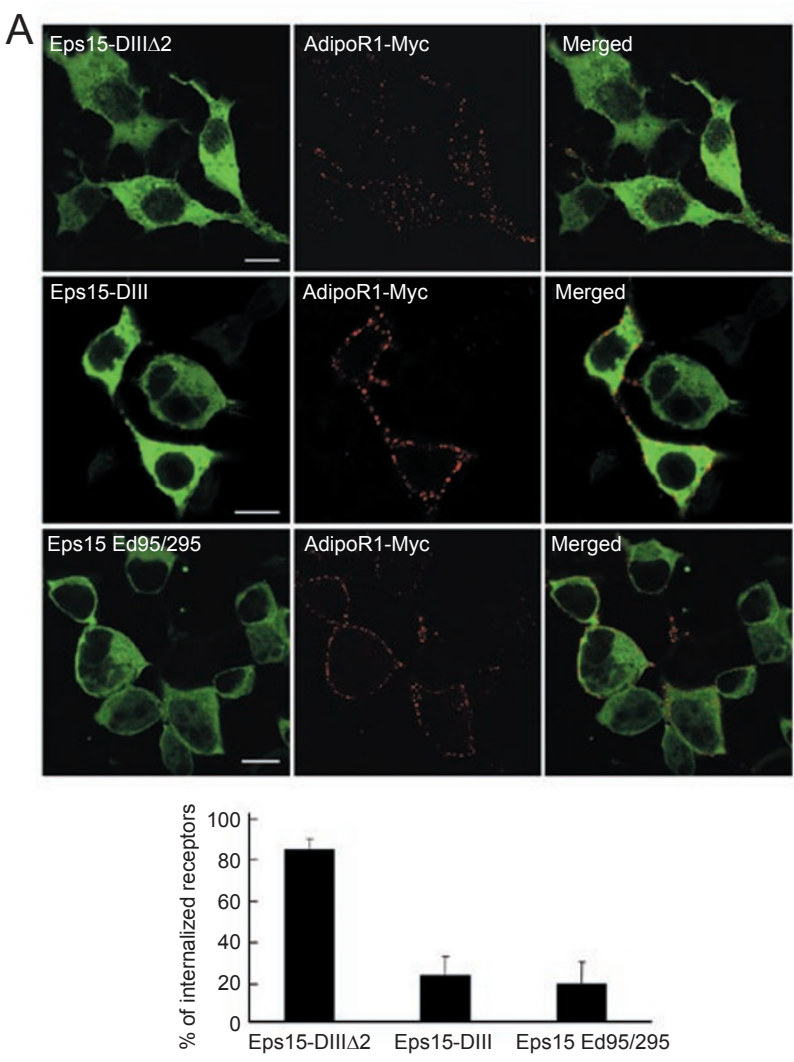

C
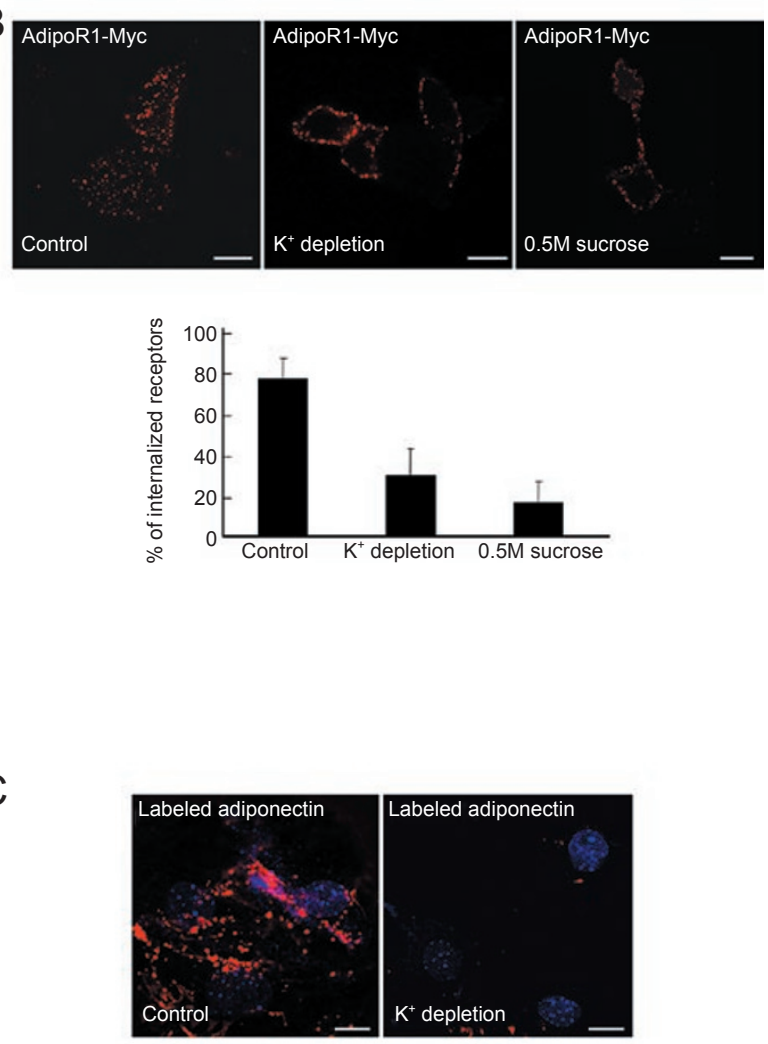

Figure 3 AdipoR1 is internalized through a clathrin-mediated pathway. (A) Endocytosis of AdipoR1 is blocked by Eps15 mutants. HEK293T cells were transfected with Myc-tagged AdipoR1 and different EGFP-fused Eps15 constructs as indicated. Membrane-bound AdipoR1 was labeled (red), and the cells were allowed to internalize at $37^{\circ} \mathrm{C}$ for 30 min before fixation and confocal imaging. The percentage of internalized receptors was quantified and is shown in the lower panel as mean \pm SD. The scale bar indicates $10 \mu \mathrm{m}$. (B) AdipoR1 internalization is blocked by $\mathrm{K}^{+}$depletion and hypertonic treatment. HEK293T cells were transfected with Myc-tagged AdipoR1. The cells were incubated in either $\mathrm{K}^{+}$depletion or hypertonic media as indicated. Membrane-bound AdipoR1 was labeled and then subjected to endocytosis at $37^{\circ} \mathrm{C}$ for $30 \mathrm{~min}$. The percentage of internalized receptors is shown in the lower panel as mean \pm SD. The scale bar indicates $10 \mu \mathrm{m}$. (C) Endocytosis of adiponectin is blocked by $\mathrm{K}^{+}$depletion. Differentiated $\mathrm{C} 2 \mathrm{C} 12$ cells were serum-starved for $2 \mathrm{~h}$ and then incubated in $\mathrm{K}+$ depletion buffer. The cells were next incubated with $10 \mu \mathrm{g} / \mathrm{ml}$ of labeled adiponectin (red) for $2 \mathrm{~h}$. Then, cells were washed to remove membrane-bound adiponectin before imaging by confocal microscopy. The scale bar indicates $10 \mu \mathrm{m}$.

(Supplementary information, Figure S2); however, globular adiponectin colocalizes little with either transferrin or transferrin receptor (Supplementary information, Figure S2), indicating that globular adiponectin is not internalized through a clathrin-dependent pathway. The different behaviors of full-length vs globular adiponectin may also indicate that the observed endocytosis of adiponectin is regulated in a specific manner.

We next investigated the endocytosis of AdipoR1. In HEK293T cells, plasma membrane-bound Myc-tagged AdipoR1 was first labeled at $4{ }^{\circ} \mathrm{C}$. Alexa 488 -labeled transferrin was added and allowed to adhere to the cell membrane for $30 \mathrm{~min}$ at $4{ }^{\circ} \mathrm{C}$. Then, the cells were incubated at $37^{\circ} \mathrm{C}$ for $10 \mathrm{~min}$ to allow for endocytosis. As shown in Figure 2D, a large portion of internalized receptors co- localizes with transferrin. Meanwhile, we found that the internalized receptors colocalize little with caveolin (Figure 2E). Therefore, these data indicate that AdipoR1 is also internalized through a clathrin-mediated pathway.

AdipoR1 endocytosis through clathrin-mediated pathway

To provide further evidence that AdipoR 1 is internalized through a clathrin-mediated pathway, we next assessed AdipoR1 endocytosis while inhibiting clathrin-mediated endocytosis. The Eps15 protein is constitutively associated with the clathrin adaptor protein complex AP-2 on the plasma membrane and is involved in clathrin-mediated endocytosis [23]. Two Eps 15 mutants, Eps15-DIII (but not the inactive variant Eps15-DIII $\Delta 2$ ) and Eps15 Ed95/295, interfere with clathrin by binding to the AP-2 adaptor and 
specifically inhibit clathrin-mediated endocytosis [23]. Myc-tagged AdipoR1 was coexpressed with the Eps15 mutant proteins in HEK293T cells. As shown in Figure 3A, AdipoR1 endocytosis was almost completely abrogated by the expression of Eps15-DIII or Eps15 Ed95/295 but was not affected by Eps15-DIII $\Delta 2$ expression. The quantification of the fluorescence signals showed that the percentage of internalized receptors dropped from $\sim 80 \%$ to $\sim 20 \%$ when Eps15-DIII or Eps15 Ed95/295 was coexpressed with AdipoR1. $\mathrm{K}^{+}$depletion and the use of a hypertonic medium ( $0.5 \mathrm{M}$ sucrose) have been reported to prevent clathrin lattice assembly and inhibit clathrin-mediated endocytosis [24-26]. We analyzed the effect of $\mathrm{K}^{+}$depletion and a hypertonic medium on AdipoR 1 endocytosis (Figure $3 \mathrm{~B})$ and found that both treatments were able to severely block the internalization of AdipoR1, with the percentage of internalized receptors dropping from about $\sim 80 \%$ to $\sim 30 \%$ with $\mathrm{K}^{+}$depletion or to $\sim 20 \%$ with the hypertonic treatment. Moreover, we found that much less adiponectin was internalized in $\mathrm{C} 2 \mathrm{C} 12$ cells after $\mathrm{K}^{+}$depletion treatment in comparison with the control cells (Figure 3C). Taken together, these results indicate that the internalization of AdipoR1 as well as adiponectin is mediated mainly through a clathrin-dependent pathway.
Rab5 is involved in the endocytosis of AdipoR1

Rab proteins make up a large family of GTPases that regulate vesicle transport in cells [27]. Rab5 is a marker of the early endosome and plays an important role in clathrin-mediated endocytosis [28]. To analyze whether Rab5 is involved in the endocytosis of AdipoR1, we coexpressed AdipoR1 and EGFP-Rab5 in Hela cells and C2C12 cells. We found that the AdipoR1 and Rab5 partly colocalize in cytoplasmic structures in both cell lines (Figure 4A and 4B). To further address the significance of Rab5 in the endocytosis of AdipoR1, we used a dominant-negative mutant of Rab5, Rab5 (S34N). We found that the endocytosis of AdipoR1 is almost completely blocked by Rab5(S34N), with $\sim 80 \%$ internalized receptors in the presence of wild-type Rab5 and less than $20 \%$ when Rab5(S34N) is coexpressed with AdipoR1 (Figure 4C). These data, therefore, suggest that Rab5 is critical for AdipoR1 endocytosis.

\section{Inhibition of AdipoR1 endocytosis enhances adiponectin-} induced AMPK phosphorylation

We next investigated how AdipoR1 endocytosis functions to regulate adiponectin signaling. Differentiated $\mathrm{C} 2 \mathrm{C} 12$ cells were pretreated with $\mathrm{K}^{+}$depletion buffer to
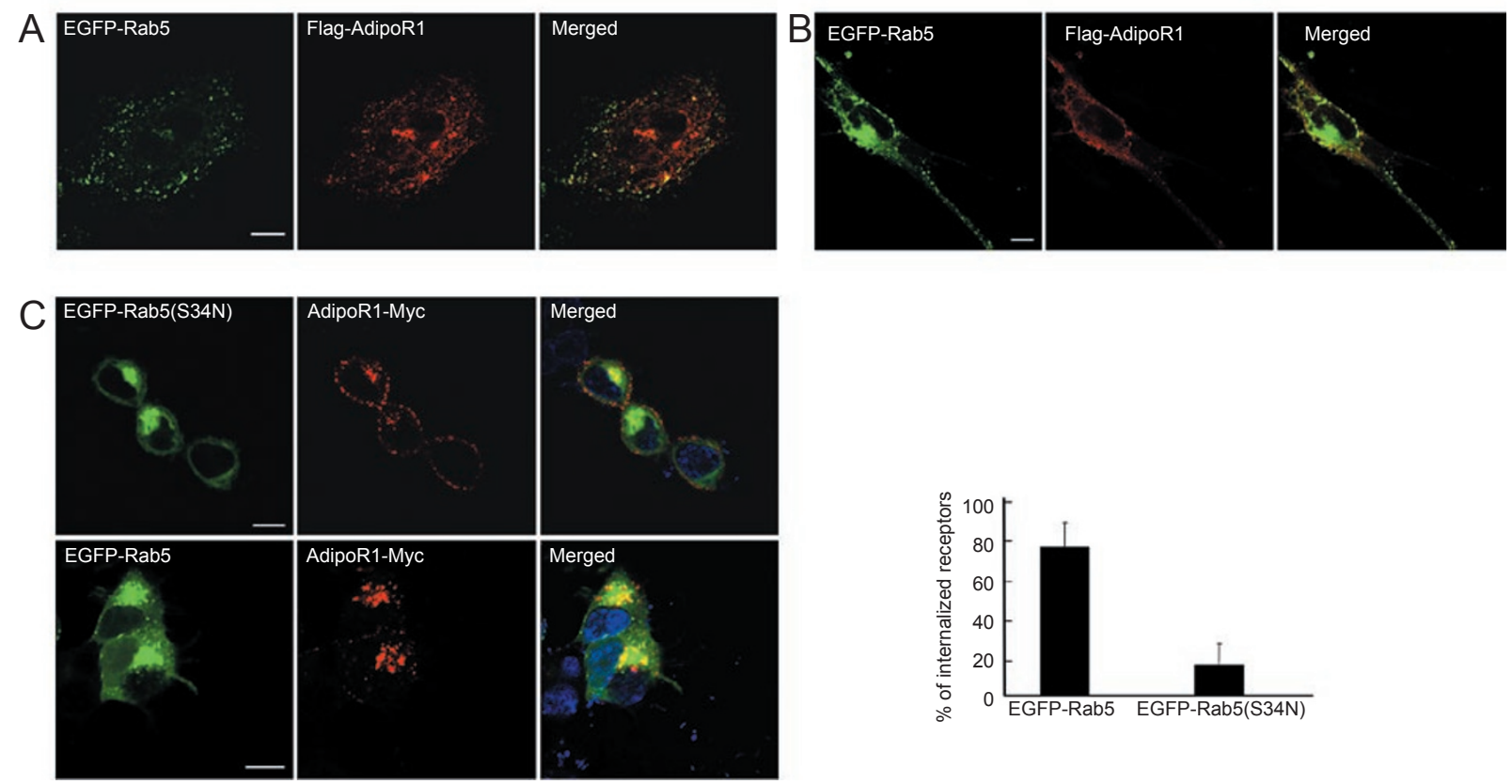

Figure 4 Rab5 is involved in AdipoR1 internalization. (A) AdipoR1 colocalizes with Rab5. Hela cells were transfected with Flagtagged AdipoR1 and EGFP-fused Rab5 as indicated. At $48 \mathrm{~h}$ after transfection, the cells were subjected to immunostaining with an anti-Flag antibody and a secondary antibody (red), followed by confocal microscopy. The scale bar indicates $10 \mu \mathrm{m}$. (B) Colocalization of AdipoR1 with Rab5 in C2C12 cells. The same experiment as described in (A) was performed in C2C12 cells. The scale bar indicates $10 \mu \mathrm{m}$. (C) Endocytosis of AdipoR1 is partly blocked by dominant negative Rab5. HEK293T cells were transfected with Myc-tagged AdipoR1 and EGFP-Rab5 or EGFP-Rab5(S34N) as indicated. Membrane-bound AdipoR1 was labeled, and the cells were allowed to internalize at $37^{\circ} \mathrm{C}$ for 30 min before fixation and confocal analysis. Percentage of internalized receptors was quantified and is shown as mean \pm SD. The scale bar indicates $10 \mu \mathrm{m}$. 
A

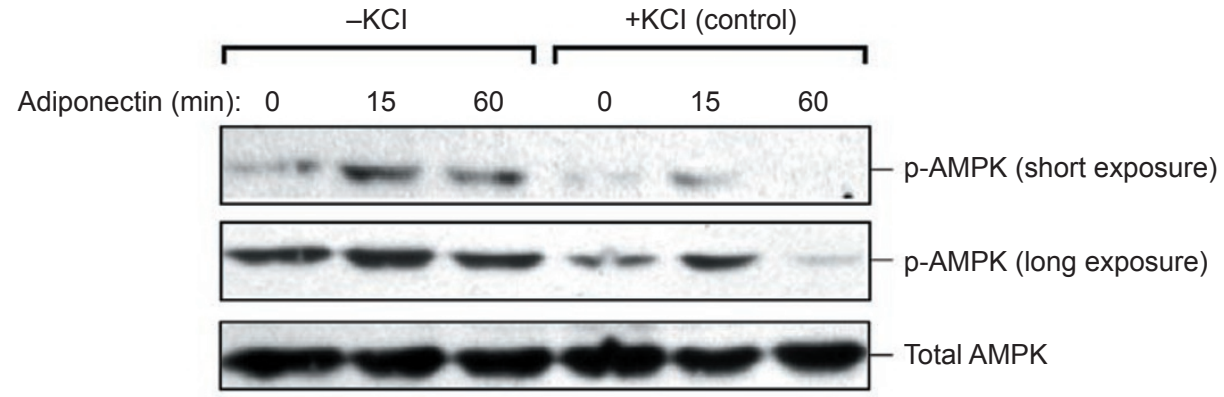

B

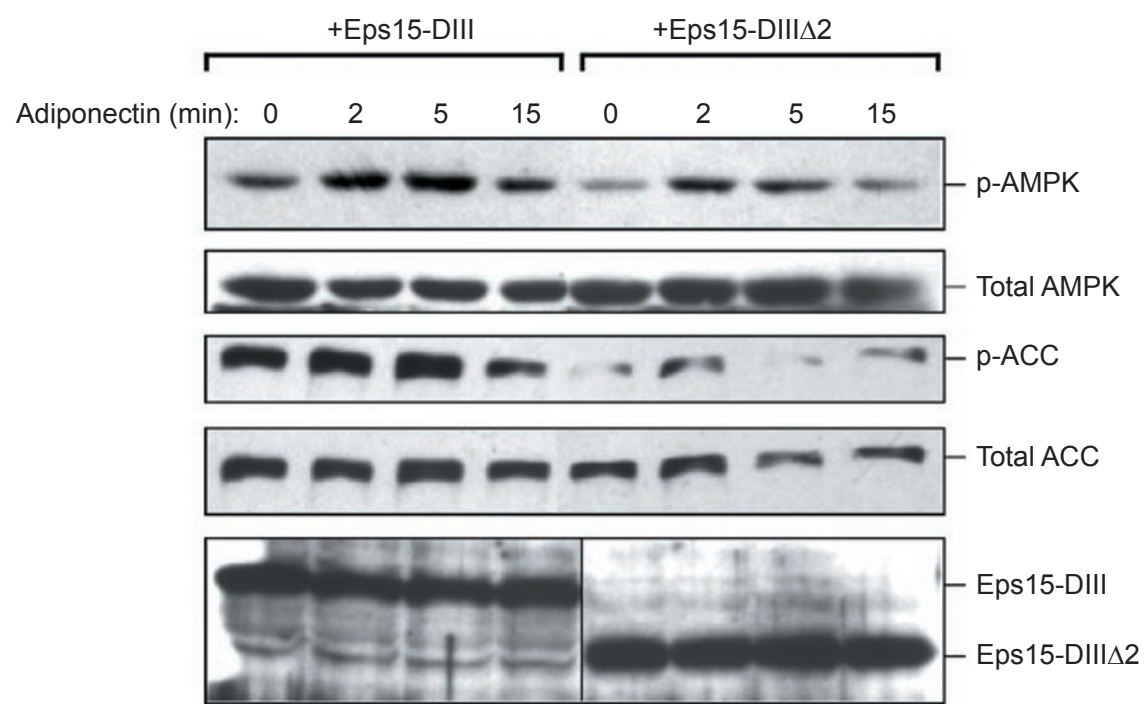

Figure 5 Inhibition of AdipoR1 endocytosis enhances adiponectin-induced AMPK phosphorylation. (A) Differentiated C2C12 cells were incubated with or without $\mathrm{K}^{+}$depletion medium, followed by treatment with $10 \mu \mathrm{g} / \mathrm{ml}$ adiponectin for various times as indicated. The cells were harvested, and the cell lysate was used for immunoblotting with antibodies for phosphorylated or total AMPK as indicated. The same experiments were performed twice with identical results. One representative result is shown. (B) HepG2 cells were transiently transfected with EGFP-Eps15-DIII and EGFP-Eps15-DIII 2 for $48 \mathrm{~h}$ and serum-starved for $24 \mathrm{~h}$ before treatment with $10 \mu \mathrm{g} / \mathrm{ml}$ adiponectin for various times as indicated. The cells were harvested, and the cell lysate was used for immunoblotting with antibodies for phosphorylated or total AMPK and ACC as indicated.

prevent the endocytosis of adiponectin receptors. The cells were then treated with $10 \mu \mathrm{g} / \mathrm{ml}$ adiponectin for different lengths of time, and the phosphorylation of AMPK was analyzed. As shown in Figure 5, adiponectin induces a sharp increase of AMPK phosphorylation, with a peak at $15 \mathrm{~min}$ in control cells, which is similar to what has been observed previously [3]. The activation of AMPK declined to a level even lower than the basal level at 60 min after stimulation [29]; however, when $\mathrm{C} 2 \mathrm{C} 12$ myotubes were treated with $\mathrm{K}^{+}$depletion buffer, adiponectin-induced phosphorylation of AMPK significantly increased and the stimulation lasted to $60 \mathrm{~min}$ after ligand addition (Figure 5A). Therefore, the inhibition of AdipoR1 endocytosis appears to enhance adiponectin-induced AMPK phosphorylation. To provide additional evidence that the inhibition of endocytosis is able to enhance adiponectin signaling, we next blocked AdipoR1 endocytosis by overexpressing Eps15-DIII in HepG2 cells, another cell line that is commonly used to investigate adiponectin signaling. Our previous result indicates that Eps15-DIII could specifically reduce the endocytosis of AdipoR1 (Figure 3A). In comparison with Eps15-DIII $\Delta 2$, a negative control, the overexpression of Eps15-DIII significantly increases both the basal and adiponectin-stimulated phosphorylation of AMPK and acetyl-coenzyme A carboxylase (ACC), another downstream target of adiponectin signaling (Figure 5B). Our data, therefore, 
indicate that endocytosis of AdipoR1 may provide a way to modulate the quantity of surface receptors and thus regulate the adiponectin signaling pathway.

\section{Discussion}

In this study, we provided evidence that adiponectin and AdipoR1 are internalized. We found that AdipoR1 endocytosis is ligand-independent and follows a clathrin-mediated pathway. Our initial characterization also suggests that Rab5 is involved in the endocytosis of AdipoR1. Furthermore, when endocytosis is blocked by $\mathrm{K}^{+}$depletion or Eps15DIII overexpression, there is significantly higher AMPK phosphorylation stimulated by adiponectin, indicating that the endocytosis of AdipoR1 might quantitatively modulate the amount of AdipoR1 on the cell surface and subsequently regulate the downstream signaling pathway.

Our study indicates that the endocytosis of AdipoR1 is constitutive. The internalization of cell surface receptors can be either constitutive or ligand-activated. In the latter case, endocytosis is initiated by post-translational modifications, such as phosphorylation or ubiquitination of the receptors upon ligand binding. On the other hand, the constitutive pathway contributes to the homeostasis of other surface receptors; it applies mainly to receptors that undergo continuous internalization and recycling. The number of surface receptors is predicated to regulate the strength and duration of downstream signaling. For example, an increase in surface receptor density could lead to sustained ERK signaling [30]. The overexpression of EGFRs in PC12 cells is able to switch the otherwise transient activation of ERK signaling upon EGF stimulation to a prolonged ERK activation and cell differentiation [31]. In our experiments, we found that blocking AdipoR1 endocytosis leads to enhanced and sustained stimulation of AMPK upon adiponectin treatment. Therefore, the endocytosis of AdipoR 1 may function as a means to modulate the quantity of surface receptors and thus regulate the strength and duration of downstream signaling.

A large group of receptors undergo clathrin-mediated endocytosis. It is generally believed that the selective recruitment of transmembrane proteins (cargo) by clathrinmediated endocytosis is initiated by the binding of specific sequences in the cytoplasmic domain of cargo proteins to the AP-2 adaptor and possibly other coat-associated proteins [32]. One specific sequence is the di-leucine motif (DE) xxxLL(I) (in which the residue at position four or, less frequently, five upstream of the first leucine is acidic, $x$ is any amino acid and the second leucine can be isoleucine). Interestingly, we found a possible di-leucine motif (KDNDYLL starting at the 106th position) in the $\mathrm{N}$-terminus of AdipoR1; this motif is in the cytoplasmic tail of the receptor and adjacent to the first transmembrane domain. Whether this sequence is involved in clathrin-mediated endocytosis of AdipoR1 needs further investigation.

Rab proteins are small GTPases that regulate vesicle docking and fusion during vesicular transport in endocytosis and exocytosis [27]. Activated Rab5 is important for sequestering ligands into clathrin-coated pits and the subsequent fusion of vesicles with early endosomes [33]. Rab5 is involved in the transport of several receptors. The expression of constitutively active Rab5 (Q79L) causes ligand-independent EGFR internalization, while the expression of dominant negative Rab5 (S34N) blocks EGF-stimulated and receptor-mediated endocytosis, which affects EGF activation in the Raf-Erk kinase pathway [34-36]. Rab5 was also found to be involved in insulin receptor-mediated endocytosis [37]. In our study, we found that AdipoR1 colocalizes with Rab5. More importantly, the dominant negative form of Rab5 is able to block the endocytosis of AdipoR1, suggesting that Rab5 regulates the internalization of AdipoR1. Recently, APPL1 was identified as a downstream adaptor of AdipoR1 [6]. Interestingly, APPL1 was previously found to be an effector of Rab5 [38, 39]. Furthermore, it has been reported that adiponectin stimulates the interaction between APPL1 and Rab5. Because APPL1 only binds to activated Rab5, these findings suggest that adiponectin may stimulate Rab5 activation. The overexpression of dominant negative Rab5 (S34N) blocks adiponectin-stimulated GLUT4 membrane translocation and p38 MAPK phosphorylation $[6,40]$. Together, these data suggest that Rab5 is involved in the regulation of signaling pathways downstream of adiponectin receptors. Our data indicate that, in addition to interacting with APPL1, Rab5 is involved in AdipoR1 internalization. It remains to be determined whether APPL1 is also involved in the endocytosis of AdipoR1. Nevertheless, our study establishes the endocytosis of adiponectin receptors as a newly identified mechanism for regulating adiponectin signaling.

\section{Materials and Methods}

\section{Cell lines, antibodies and plasmids}

HEK293T, Hela and C2C12 cells were grown in DMEM media supplemented with $10 \%$ fetal bovine serum (FBS), $100 \mu \mathrm{g} / \mathrm{ml}$ penicillin and $100 \mu \mathrm{g} / \mathrm{ml}$ streptomycin (all from GIBCO-BRL, Gaithersburg, MD, USA). Cells were incubated at $37^{\circ} \mathrm{C}$ in humidified air with $5 \%$ $\mathrm{CO}_{2}$ and subcultured every 2 or 3 days. Differentiation of $\mathrm{C} 2 \mathrm{C} 12$ cells was induced by culturing in $2 \%$ horse serum-containing DMEM, which was changed daily for 5-7 days. Transfection was performed by the PEI method [41].

The anti-FLAG antibody was from Sigma-Aldrich (Saint Louis, MO, USA) and the anti-Myc antibody was from Santa Cruz Biotechnology (Santa Cruz, CA, USA). The antibody for transferrin receptor was from USCNLIFE Company (Wuhan, China). The antibodies for 
AMPK, phosphorylated AMPK, ACC and phosphorylated ACC were from Cell Signaling Technology (Danvers, MA, USA), and caveolin1 antibody was from Abcam (Cambridge, UK). Human Alexa Fluor 488-conjugated transferrin and Alexa Fluor 555 Microscale Protein labeling kit was from Invitrogen-Molecular Probes (Carlsbad, CA, USA). Human recombinant full-length adiponectin was from R\&D system (Minneapolis, MN, USA).

The human AdipoR1 cDNA was cloned by PCR with human liver cDNA as the template with the following primers: $5^{\prime}$-AGG GTA TTG AGG TAC CAG CCA GAT G-3' and 5'-GGA AGG CTC AGA GAA GGG TGT CAT C-3'. For Flag-tagged AdipoR1, AdipoR1 cDNA was cloned into pRc/CMV (Invitrogen), and a FLAG tag was added into the $\mathrm{N}$-terminus by PCR. The AdipoR1 cDNA was cloned into pCDNA3.1/myc-His (Invitrogen) to include a Myc tag at the C-terminus. Human Rab5 cDNA was cloned by PCR with HepG2 cell cDNA as the template with the following primers: 5'-GCT AGT CGA GGC GCAACA-3' and 5'-TTA GTT ACT ACAACA CTG-3'. The PCR fragment was cloned into the $\mathrm{pEGFP}-\mathrm{C} 1$ vector (Clontech, Mountain View, CA, USA). GFP-Rab5S34N was generated by sitedirected mutagenesis PCR and verified by DNA sequencing. The plasmids pEGFP Eps15-DIII, Eps15-DIII 22 and Eps15 Ed95/295 were kindly provided by Alexandre Benmerah [23].

\section{Endocytosis analysis of adiponectin}

Full-length and globular adiponectin were labeled with the Alexa Fluor 555 Microscale Protein labeling kit (Invitrogen-Molecular Probes) following the manufacturer's instruction. For analysis of adiponectin internalization in Hela cells, cells were serum-starved for $24 \mathrm{~h}$ and then incubated with $10 \mu \mathrm{g} / \mathrm{ml}$ of labeled adiponectin with or without 20-fold unlabeled adiponectin in DMEM for $2 \mathrm{~h}$. The surface-bound adiponectin was removed with three washes with ice-cold acid wash buffer $(150 \mathrm{mM} \mathrm{NaCl}$ and $20 \mathrm{mM} \mathrm{Na}$-citrate at $\mathrm{pH}$ 5.0). The cells were then fixed with $4 \%$ paraformaldehyde and imaged by confocal microscopy. For colocalization of transferrin and adiponectin, differentiated C2C12 cells were serum-starved for $2 \mathrm{~h}$ and then incubated with $50 \mu \mathrm{g} / \mathrm{ml}$ transferrin and $10 \mu \mathrm{g} / \mathrm{ml}$ labeled full-length adiponectin or $2 \mu \mathrm{g} / \mathrm{ml}$ labeled globular adiponectin in DMEM for $2 \mathrm{~h}$ or $10 \mathrm{~min}$. The surface-bound transferrin and adiponectin were removed with three washes of ice-cold acid wash buffer; cells were then fixed and imaged. For colocalization studies with transferrin receptors, adiponectin was internalized for $10 \mathrm{~min}$ before immunostaining and analyzing with confocal microscopy.

\section{Analysis of receptor internalization}

AdipoR1 internalization studies were carried out as described previously [14]. Briefly, membrane-bound Myc-tagged AdipoR1 was detected by incubating cells with an anti-Myc antibody in a buffer containing $20 \mathrm{mM}$ HEPES (pH 7.5), $140 \mathrm{mM} \mathrm{NaCl}, 1 \mathrm{mM}$ $\mathrm{CaCl}_{2}, 1 \mathrm{mM} \mathrm{MgSO} 4,10 \mathrm{mM} \mathrm{KCl}, 5.5 \mathrm{mM}$ glucose and $0.5 \% \mathrm{BSA}$ for $2 \mathrm{~h}$ at $4{ }^{\circ} \mathrm{C}$. After cells had been washed three times with icecold PBS buffer, they were incubated with Alexa-546 secondary antibody for $1 \mathrm{~h}$ at $4{ }^{\circ} \mathrm{C}$. Cells were then washed and incubated in complete medium (DMEM $+10 \% \mathrm{FBS})$ at $37{ }^{\circ} \mathrm{C}$ for the indicated times. The cells were fixed and analyzed by confocal microscopy. To label only the membrane-localized but not internalized receptors, a slightly different protocol was used. Briefly, after labeling the membrane-bound AdipoR1 by primary anti-Myc antibody at $4{ }^{\circ} \mathrm{C}$, endocytosis was initiated by an incubation at $37{ }^{\circ} \mathrm{C}$ for different times, followed by fixation and staining with Alexa-546 secondary antibody without detergent treatment. For colocalization studies of transferrin and internalized AdipoR1, membrane-bound AdipoR1myc was labeled as indicated, and then $50 \mu \mathrm{g} / \mathrm{ml}$ of transferrin was allowed to adhere to cell membranes at $4{ }^{\circ} \mathrm{C}$ for 30 min before being subjected to internalization for $10 \mathrm{~min}$ at $37^{\circ} \mathrm{C}$.

\section{$K^{+}$depletion and hypertonic sucrose treatment}

Clathrin-dependent endocytosis was abrogated by $\mathrm{K}^{+}$depletion and a hypertonic sucrose treatment. Briefly, HEK293T or differentiated $\mathrm{C} 2 \mathrm{C} 12$ cells were incubated in DMEM:water (1:1) for $5 \mathrm{~min}$ at $37^{\circ} \mathrm{C}$ and then incubated in a buffer $(20 \mathrm{mM}$ HEPES at $\mathrm{pH} 7.5$, $140 \mathrm{mM} \mathrm{NaCl}, 1 \mathrm{mM} \mathrm{CaCl}_{2}, 1 \mathrm{mM} \mathrm{MgSO}_{4}, 5.5 \mathrm{mM}$ glucose and $0.5 \% \mathrm{BSA}$ ) for $1 \mathrm{~h}$ at $37{ }^{\circ} \mathrm{C}$. Control cells were incubated in the buffer supplemented with $10 \mathrm{mM} \mathrm{KCl}$. HEK293T cells were treated with $0.5 \mathrm{M}$ sucrose dissolved in DMEM for $30 \mathrm{~min}$ of hypertonic medium treatment.

\section{Confocal immunofluorescence microscopy}

Cells were plated on glass coverslips in a 12 -well plate and allowed to adhere overnight. They were then transfected with the indicated plasmids and incubated for $48 \mathrm{~h}$. Slides were fixed for 10 min in $4 \%$ formaldehyde, washed extensively with PBS, permeabilized with $0.1 \%$ Triton X-100 for 10 min and blocked for $1 \mathrm{~h}$ in $3 \%$ BSA in PBS. Slides were then sequentially incubated with primary antibodies, washed with PBS, incubated for $1 \mathrm{~h}$ with Alexa 488- or Alexa 546-conjugated secondary antibodies (Invitrogen-Molecular Probes). The nuclei were counterstained with DAPI for $5 \mathrm{~min}$. Cells were mounted with an anti-fade mounting medium (Biomeda, Foster City, CA, USA), and immunofluorescence was visualized with a confocal microscope (LSM 510, Zeiss, Germany). To quantify internalized receptors, two circles - one outlining the whole cell and the other beneath the plasma membrane - were drawn manually. After the background was subtracted, the fluorescence intensities in each circle were measured by Image-Pro Plus 5.02 and counted as wholecell and intracellular fluorescence. The percentages of internalized receptors were calculated by dividing the intracellular fluorescence by whole-cell fluorescence. At least 50 cells were quantified for each experimental group.

\section{Western blotting analysis}

For Western blotting analysis, the cells were lysed in RIPA buffer (150 mM NaCl, 1\% Triton X-100, 0.5\% sodium deoxycholate, $0.1 \%$ SDS, $50 \mathrm{mM}$ Tris- $\mathrm{HCl}$ at $\mathrm{pH}$ 7.4) containing phosphatase inhibitors and a protease inhibitor cocktail (Sigma-Aldrich). The lysate was subjected to SDS-PAGE, transferred to PVDF membranes and incubated with the primary antibodies followed by horseradish peroxidase-conjugated secondary antibody (Amersham, Buckinghamshire, UK). The bound antibody was visualized using enhanced chemiluminescence from Pierce (Rockford, IL, USA).

\section{Acknowledgments}

We wish to thank Dr Alexandre Benmerah (Institut Cochin, Département Maladies Infectieuses Paris F-75014, France) for providing the plasmids. This work was supported by research grants from the Chinese Academy of Sciences (One Hundred Talents Program and the Knowledge Innovation Program KSCX1-YW-02), the National Natural Science Foundation of China (30588002), Science \& Tech- 
nology Commission of Shanghai Municipality (06XD14022 and 07DJ14005), and the Ministry of Science and Technology of China (2006CB503900 and 2007CB947100) to Yan Chen.

\section{References}

1 Spiegelman BM, Flier JS. Obesity and the regulation of energy balance. Cell 2001; 104:531-543.

2 Kadowaki T, Yamauchi T, Kubota N, et al. Adiponectin and adiponectin receptors in insulin resistance, diabetes, and the metabolic syndrome. J Clin Invest 2006; 116:1784-1792.

3 Yamauchi T, Kamon J, Ito Y, et al. Cloning of adiponectin receptors that mediate antidiabetic metabolic effects. Nature 2003; 423:762-769.

4 Yamauchi T, Nio Y, Maki T, et al. Targeted disruption of AdipoR1 and AdipoR2 causes abrogation of adiponectin binding and metabolic actions. Nat Med 2007; 13:332-339.

5 Kadowaki T, Yamauchi T. Adiponectin and adiponectin receptors. Endocr Rev 2005; 26:439-451.

6 Mao X, Kikani CK, Riojas RA, et al. APPL1 binds to adiponectin receptors and mediates adiponectin signalling and function. Nat Cell Biol 2006; 8:516-523.

7 Tsao P, Cao T, von Zastrow M. Role of endocytosis in mediating downregulation of G-protein-coupled receptors. Trends Pharmacol Sci 2001; 22:91-96.

8 Tsao PI, von Zastrow M. Type-specific sorting of G protein-coupled receptors after endocytosis. J Biol Chem 2000; 275:1113011140.

9 Carman CV, Benovic JL. G-protein-coupled receptors: turn-ons and turn-offs. Curr Opin Neurobiol 1998; 8:335-344.

10 Goodman OB Jr, Krupnick JG, Santini F, et al. Beta-arrestin acts as a clathrin adaptor in endocytosis of the beta2-adrenergic receptor. Nature 1996; 383:447-450.

11 Lefkowitz RJ, Pitcher J, Krueger K, Daaka Y. Mechanisms of beta-adrenergic receptor desensitization and resensitization. $A d v$ Pharmacol 1998; 42:416-420.

12 Sorkin A. Internalization of the epidermal growth factor receptor: role in signalling. Biochem Soc Trans 2001; 29 (Pt 4):480-484.

13 Ehrlich M, Shmuely A, Henis YI. A single internalization signal from the di-leucine family is critical for constitutive endocytosis of the type II TGF-beta receptor. J Cell Sci 2001; 114 (Pt 9):17771786.

14 Di Guglielmo GM, Le Roy C, Goodfellow AF, Wrana JL. Distinct endocytic pathways regulate TGF-beta receptor signalling and turnover. Nat Cell Biol 2003; 5:410-421.

15 Hayes S, Chawla A, Corvera S. TGF beta receptor internalization into EEA1-enriched early endosomes: role in signaling to Smad2. J Cell Biol 2002; 158:1239-1249.

16 Mousavi SA, Malerod L, Berg T, Kjeken R. Clathrin-dependent endocytosis. Biochem J 2004; 377 (Pt 1):1-16.

17 Le Roy C, Wrana JL. Clathrin- and non-clathrin-mediated endocytic regulation of cell signalling. Nat Rev Mol Cell Biol 2005; 6:112-126.

18 Ferguson SS. Evolving concepts in G protein-coupled receptor endocytosis: the role in receptor desensitization and signaling. Pharmacol Rev 2001; 53:1-24.

19 Hotta K, Funahashi T, Arita Y, et al. Plasma concentrations of a novel, adipose-specific protein, adiponectin, in type 2 diabetic patients. Arterioscler Thromb Vasc Biol 2000; 20:1595-1599.
20 Tang YT, Hu T, Arterburn M, et al. PAQR proteins: a novel membrane receptor family defined by an ancient 7-transmembrane pass motif. J Mol Evol 2005; 61:372-380.

21 Hanover JA, Willingham MC, Pastan I. Kinetics of transit of transferrin and epidermal growth factor through clathrin-coated membranes. Cell 1984; 39 ( Pt 1):283-293

22 Silva W, Maldonado H, Chompre G, Mayol N. Caveolae a new subcellular transport organelle. Bol Assoc Med P R 1998; 90:3033.

23 Benmerah A, Bayrou M, Cerf-Bensussan N, Dautry-Varsat A. Inhibition of clathrin-coated pit assembly by an Eps 15 mutant. J Cell Sci 1999; 112 (Pt 9):1303-1311.

24 Larkin JM, Brown MS, Goldstein JL, Anderson RG. Depletion of intracellular potassium arrests coated pit formation and receptormediated endocytosis in fibroblasts. Cell 1983; 33:273-285.

25 Daukas G, Zigmond SH. Inhibition of receptor-mediated but not fluid-phase endocytosis in polymorphonuclear leukocytes. J Cell Biol 1985; 101 (Pt 1):1673-1679.

26 Heuser JE, Anderson RG. Hypertonic media inhibit receptormediated endocytosis by blocking clathrin-coated pit formation. J Cell Biol 1989; 108:389-400.

27 Somsel Rodman J, Wandinger-Ness A. Rab GTPases coordinate endocytosis. J Cell Sci 2000; 113 (Pt 2):183-192.

28 McLauchlan H, Newell J, Morrice N, et al. A novel role for Rab5-GDI in ligand sequestration into clathrin-coated pits. Curr Biol 1998; 8:34-45.

29 Yamauchi T, Kamon J, Minokoshi Y, et al. Adiponectin stimulates glucose utilization and fatty-acid oxidation by activating AMPactivated protein kinase. Nat Med 2002; 8:1288-1295.

30 Murphy LO, Blenis J. MAPK signal specificity: the right place at the right time. Trends Biochem Sci 2006; 31:268-275.

31 Traverse S, Seedorf K, Paterson H, et al. EGF triggers neuronal differentiation of $\mathrm{PC} 12$ cells that overexpress the EGF receptor. Curr Biol 1994; 4:694-701.

32 Sorkin A. Cargo recognition during clathrin-mediated endocytosis: a team effort. Curr Opin Cell Biol 2004; 16:392-399.

33 Li G. Rab5 GTPase and endocytosis. Biocell 1996; 20:325330.

34 Barbieri MA, Fernandez-Pol S, Hunker C, Horazdovsky BH, Stahl PD. Role of rab5 in EGF receptor-mediated signal transduction. Eur J Cell Biol 2004; 83:305-314.

35 Dinneen JL, Ceresa BP. Continual expression of Rab5(Q79L) causes a ligand-independent EGFR internalization and diminishes EGFR activity. Traffic 2004; 5:606-615.

36 Barbieri MA, Roberts RL, Gumusboga A, et al. Epidermal growth factor and membrane trafficking. EGF receptor activation of endocytosis requires Rab5a. J Cell Biol 2000; 151:539-550.

37 Hunker CM, Kruk I, Hall J, et al. Role of Rab5 in insulin receptor-mediated endocytosis and signaling. Arch Biochem Biophys 2006; 449:130-142.

38 Miaczynska M, Christoforidis S, Giner A, et al. APPL proteins link Rab5 to nuclear signal transduction via an endosomal compartment. Cell 2004; 116:445-456.

39 Zhu G, Chen J, Liu J, et al. Structure of the APPL1 BAR-PH domain and characterization of its interaction with Rab5. Embo J 2007; 26:3484-3493

40 Cheng KK, Lam KS, Wang Y, et al. Adiponectin-induced endothelial nitric oxide synthase activation and nitric oxide production are mediated by APPL1 in endothelial cells. Diabetes 2007 
56:1387-1394.

41 Sun X, Han R, Wang Z, Chen Y. Regulation of adiponectin receptors in hepatocytes by the peroxisome proliferator-activated receptor-gamma agonist rosiglitazone. Diabetologia 2006; 49:1303-1310.

(Supplementary information is linked to the online version of the paper on the Cell Research website.) 